\title{
Surgical Repair of Descemet's Membrane Detachment Following Phacoemulsification by Intracameral Air Injection and Suture Fixation
}

\author{
Balaji Kannan, Vijayalakshmi Balaji, Kummararaj Gonvindarajan, Shereen Kummararaj \\ Dr A Govindarajan Eye Hospital, Trichy, Inda
}

Post-surgical Descemet's membrane detachment is a rare complication that has a serious visual impact if not treated adequately. This report is of a 74-year-old patient with Descemet's membrane detachment after phacoemulsification who underwent successful repair by intracameral air injection and suture fixation.

Key words: Descemet membrane, Intraocular pressure, Viscoelastic substances

Asian J Ophthalmol. 2011;12:221-3.

\section{Introduction}

Post-surgical Descemet's membrane detachment (DMD) is a rare complication that has a serious visual impact if not treated adequately. Post-surgical DMD is usually related to cataract surgery, but it has also been described after other ocular operations. ${ }^{1,2}$ At the clinical level, 2 types of DMD are differentiated: planar, when the separation between Descemet's membrane and the stroma is $<1 \mathrm{~mm}$ and non-planar when the separation exceeds $1 \mathrm{~mm} .{ }^{3}$ Small detachments caused by incisions, with minimal clinical significance, are not included in this classification. Post-surgical DMD can be recognised intraoperatively or may go unrecognised during surgery and be discovered postoperatively. Post-surgical DMD can be localised, extensive, or total, and can cause permanent corneal decompensation requiring surgical treatment. In 1992, Macsai et al reported the first patient with sight-threatening DMD associated with phacoemulsification. ${ }^{4}$ Only a few cases of DMD associated with phacoemulsification have been described. ${ }^{5}$ In most cases, DMD remains localised to the area adjacent to the corneoscleral incision. ${ }^{6}$

Management of DMD depends on the degree and location of the detachment. ${ }^{7}$ Air, viscoelastic, and expansible gases have been used for DM reattachment, but most surgical repositions were performed with air or viscoelastic. ${ }^{8}$ Another way to reattach a Descemet's membrane is by descemetopexy using through and through 10-0 sutures with long bites that include the edge of the detached Descemet's membrane. ${ }^{9}$ Penetrating keratoplasty should

Correspondence: Dr Balaji Kannan,180/72, East Chitra Street,

Srirangam Trichy, Tamil Nadu 620 006, India.

Tel: (91 431) 431 1555;

E-mail: kbalaji36@hotmail.com be the last management option. Large DMD results in persistent corneal oedema and decreased visual acuity, and requires surgical repair. In some patients with extensive DMD and corneal haze, ultrasound biomicroscopy can be used to assist the surgical repair. ${ }^{10}$ This report is of a patient with DMD after phacoemulsification who underwent successful repair.

\section{Case Report}

A 74-year-old man underwent sutureless phacoemulsification with posterior chamber lens implantation in the right eye via a stepped sclerocorneal incision located superotemporally. During the operation, an undulating DMD involving the central cornea was observed, possibly caused by inadvertent hydrodissection when performing corneal stromal hydration with balanced salt solution injection at the end of the procedure. A wave was observed in the cornea, which expanded concentrically from the main incision (Figure 1a). Manipulation with viscoelastic and tamponade with sterile intracameral air was performed to repair the DMD. On the first postoperative day there was diffuse corneal oedema in the operated eye, and the patient's visual acuity was counting finders at $3 \mathrm{~m}$. The patient was given topical steroids (prednisolone acetate 1\%) and topical antibiotics (ofloxacin $0.3 \%$ ) every hour. Topical hypertonic salt agents ( $5 \%$ sodium chloride) were given 4 hourly to reduce the corneal oedema by hygroscopic action. Two days after surgery, the corneal oedema persisted despite continued treatment. One week later, the DMD had progressed more diffusely (Figure 1b), and the patient's vision had decreased to counting fingers close to the face.

The DMD was immediately repaired by suture fixation. Four long full-thickness $10-0$ nylon sutures were used to fixate the 
Figure 1. Diffuse non-planar Descemet's detachment involving two-thirds of the cornea (arrows) (a) immediately after surgery; and (b) 1 week later.
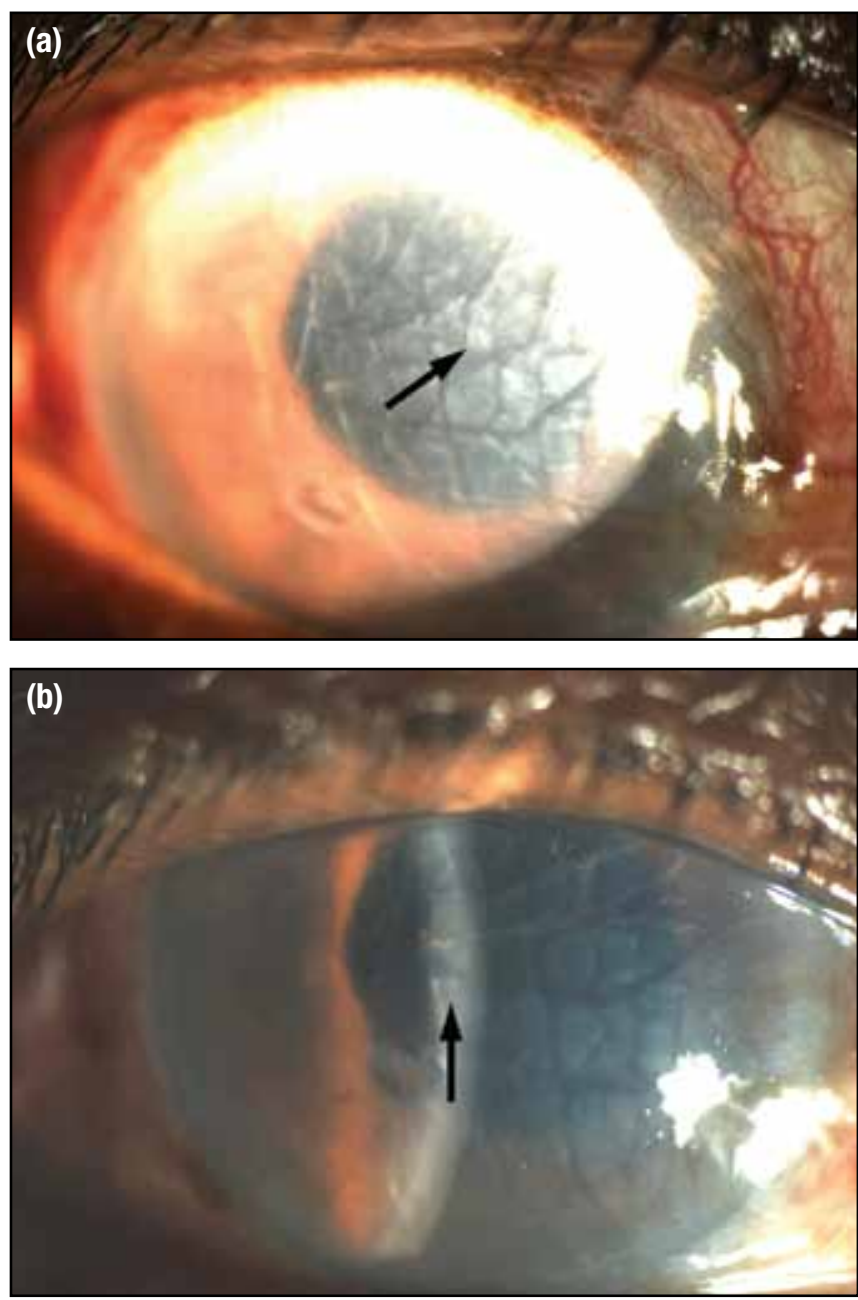

membrane to the cornea, including the internal incision of the sclerocorneal tunnel. Intracameral air injection was used to fill the anterior chamber after a side port paracentesis was performed to drain the sub-Descemet's membrane fluid. A single suture was placed at side port, and the knots were buried in the stroma. Pilocarpine was instilled immediately postoperatively to prevent air travelling through the pupil to the posterior chamber or vitreous cavity. The patient was instructed to maintain a supine position. The next day, the Descemet's membrane was completely attached, and the cornea was clear (Figure 2). After 3 months, the cornea was clear (Figure 3), the membrane remained attached, and the bestcorrected visual acuity was 20/40.

\section{Discussion}

DMD with associated corneal oedema is a rare complication following cataract surgery and may cause permanent corneal decompensation. Although spontaneous resolution of DMD has been reported, surgical intervention is often required. ${ }^{11}$ Techniques
Figure 2. Day 1 following surgical repair of detachment with an air bubble freely floating in anterior chamber.

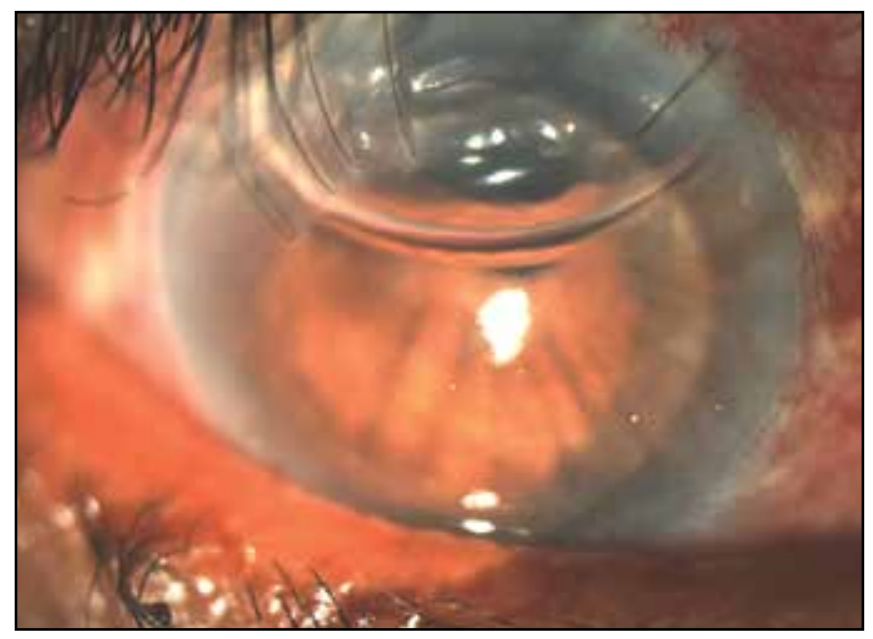

Figure 3. A completely attached Descemet's membrane during follow-up.

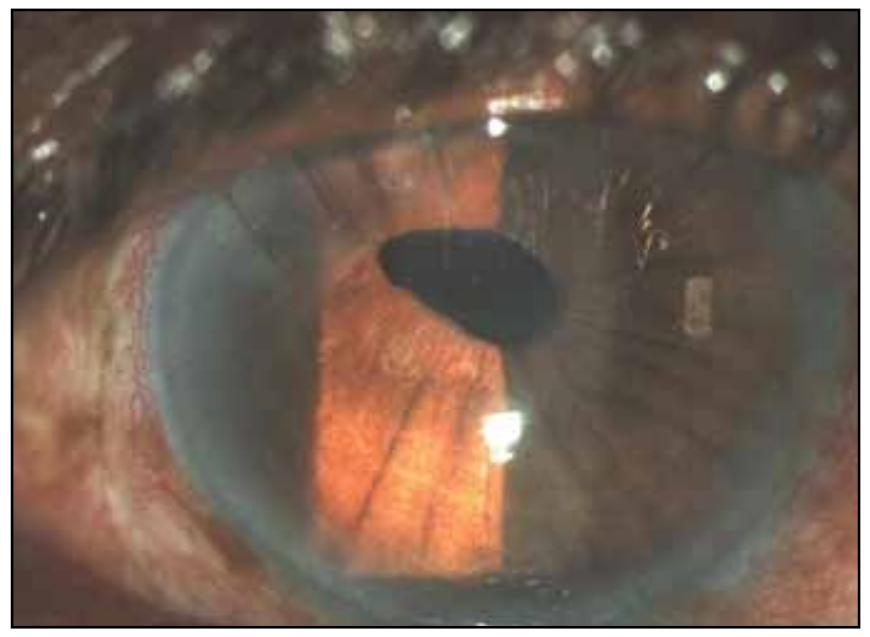

successfully used to treat intractable DMD include tamponade with $20 \%$ sulfur hexafluoride gas injected into the anterior chamber, ${ }^{8}$ or suturing of Descemet's membrane to the corneal stroma via a superior scleral wound ${ }^{5}$ or with trans-corneal polypropylene mattress sutures in combination with intracameral air injection. ${ }^{12}$

Several mechanisms have been proposed as the possible cause of DMD, including shallow chambers, complicated or repeated operations, inadvertent insertion of instruments between the corneal stroma and Descemet's membrane, anterior and shelved incisions, and the use of dull blades. ${ }^{13,14}$ Engaging the Descemet's membrane during intraocular lens implantation or with the Irrigation/aspiration device (when mistaken for an anterior capsular remnant) can also lead to extensive DMD. Some authors have reported that inadvertent injection of viscoelastic material by inserting the cannula between the Descemet's membrane and the corneal stroma may be the most common cause of DMD when using current surgical techniques. ${ }^{15}$ 
In this patient, although manipulation with viscoelastic and intracameral air injection was performed at the end of surgery, the DMD persisted because of the communication with the anterior chamber at the sclerocorneal wound. This patient responded promptly to intracameral air tamponade and simple full thickness corneal sutures through the original surgical wound, which presumably prevented further ingress of aqueous beneath the detachment. Buoyancy of the injected air bubble in the anterior chamber, along with the corneal endothelial pump action and supportive hygroscopic action of the hyperosmotic saline solution helped with reattachment of the DMD. No predisposing factors were found to influence the occurrence of DMD in this patient.

In the course of anterior segment surgery, DMD usually occurs in the entry incision, particularly when manipulated excessively. This was the case for this patient, for whom the small detachment that occurred during the surgery would have increased further during stromal hydration (with an apparently deficient cannula angle) at the end of the surgery. In the absence of special gases, air instillation into the anterior chamber is a promising intervention in the presence of a DMD. Importantly, early surgical intervention for large non-planar DMD may provide good results and faster visual recovery.

\section{References}

1. Theodore FH. Complications after cataract surgery. Int Ophthalmol Clin. 1964;4:913-48.

2. Sugar HS. Prognosis in stripping of Descemet's membrane. Am J
Ophthalmol. 1967;63:140-3

3. Mackool RJ, Holtz SJ. Descemet's membrane detachment. Arch Ophthalmol. 1977;95:459-63.

4. Macsai MS, Gainer KM, Chisholm L. Repair of Descemet's membrane detachment with perfluoropropane (C3F8). Cornea. 1998;17: 129-34.

5. Amaral CE, Palay DA. Technique for repair of Descemets membrane detachment. Am J Ophthalmol. 1999;127:88-90.

6. Marcon AS, Rapuano CJ, Jones MR, Laibson PR, Cohen EJ. Descemet's membrane detachment after cataract surgery: management and outcome. Ophthalmology. 2002;109:2325-30.

7. Hagan JC. Treatment of progressive Descemet's membrane detachment. Ophthalmic Surg. 1992;23:641.

8. Ellis DR, Cohen KL. Sulfur hexafluoride gas in the repair of Descemet's membrane detachment. Cornea. 1995;14:436-7.

9. Jeng $\mathrm{BH}$, Meisler DM. A combined technique for surgical repair of Descemet's membrane detachments. Ophthalmic Surg Lasers Imaging. 2006;37:291-7.

10. Assia El, Levkovich-Verbin H, Blumenthal M. Management of Descemet's membrane detachment. J Cataract Refract Surg. 1995; 21:714-7.

11. Minkovitz JB, Schrenk LC, Pepose JS. Photo essay: spontaneous resolution of an extensive detachment of Descemet's membrane following phacoemulsification. Arch Ophthalmol. 1994;112:551-2.

12. Vastine DW, Weinberg RS, Sugar J, Binder PS. Stripping of Descemet's membrane associated with intraocular lens implantation. Arch Ophthalmol. 1983;101:1042-5.

13. Iradier MT, Moreno E, Aranguez C, Cuevas J, Garcia Feijoo J, Garcia Sanchez J. Late spontaneous resolution of a massive detachment of Descemet's membrane after phacoemulsification. J Cataract Refract Surg. 2002;28:1071-3.

14. Anderson CJ. Gonioscopy in no-stitch cataract incisions. J Cataract Refract Surg. 1993;19:620-1.

15. Mulhern M, Barry P, Condon P. A case of Descemet's membrane detachment during phacoemulsificaton surgery. $\mathrm{Br} \mathrm{J}$ Ophthalmol. 1996;80:185-6. 